\title{
On Einstein warped product space with respect to semi symmetric metric connection
}

\author{
Buddhadev Pal*(i), Pankaj Kumar (iD) \\ Department of Mathematics, Institute of Science, Banaras Hindu University, Varanasi-221005, India
}

\begin{abstract}
In this paper, we study Einstein warped product space with respect to semi symmetric metric connection. During this study we establish some results on curvature, Ricci and scalar tensors with respect to semi symmetric metric connection and second order semi symmetric metric connection. In the last section, we investigate under what conditions, if $M$ is an Einstein warped space with nonpositive scalar curvature and compact base with respect to semi symmetric metric connection then $M$ is simply a Riemannian product space.
\end{abstract}

Mathematics Subject Classification (2020). 53C25, 53C50

Keywords. Einstein manifold, semi symmetric metric connection, warped product space, Ricci tensor, Hessian tensor, Ricci identity

\section{Introduction}

Let $\left(B, g_{B}\right)$ and $\left(F, g_{F}\right)$ be two Riemannian manifolds with a positive smooth function $f$ on $B$. The metric on the product space $B \times F$ is given by

$$
g=\pi^{*}\left(g_{B}\right)+(f \circ \pi)^{2} \sigma^{*}\left(g_{F}\right)
$$

where $\pi: B \times F \rightarrow B$ and $\sigma: B \times F \rightarrow F$ are the projections on the manifolds $B$ and $F$ respectively and ${ }^{*}$ denotes pull-back operator on tensors. The product space $B \times F$ equipped with metric tensor $g$ is called warped product space, was first introduced by $\mathrm{R}$. Bishop and B. O'Neil [2]. It is denoted by $M=B \times_{f} F$. The function $f$ is called warping function of the warped product. $B$ and $F$ are called the base and fiber of $M$ respectively. When the warping function $f$ is constant then the warped product $B \times_{f} F$ is simply a Riemannian product space. After that B. O'Nill [13], studied the geometric properties of warped product in detail.

A Riemannian manifold $\left(M^{n}, g\right),(n>2)$ is said to be an Einstein manifold if the condition $S(X, Y)=\frac{r}{n} g(X, Y)$ holds on $M$, where $S$ and $r$ denote the Ricci tensor and the scalar curvature of $\left(M^{n}, g\right)$ respectively. According to [3] the above equation is called the Einstein metric condition.

In 2002, D. S. Kim [10] and in 2003, Kim and Kim [11] discussed the results about compact Einstein warped product space. After that in 2005, Mustafa [12] extended the

\footnotetext{
*Corresponding Author.

Email addresses: pal.buddha@gmail.com (B. Pal), pankaj.kumar14@bhu.ac.in (P. Kumar)

Received: 19.06.2020; Accepted: 16.05.2021
} 
theorem of Kim and Kim. Then in [5], D. Dumitru studied the existence of compact Einstein warped products. In 2017, F. E. S. Feitosa, A. A. F. Filho and J. N. V. Gomes [7] proved that if warping function on gradient Ricci soliton warped product attains maximum and minimun then it must be Riemannian product.

The concept of a semi-symmetric linear connection on a differential manifold was introduced by Friedmann and Schouten [6]. After that in 1932, Hayden [9] and in 1970, K. Yano [16] discussed some properties of semi-symmetric metric connection. K.Yano established that a Riemannian manifold is of constant curvature iff it considers a semi-symmetric metric connection for which the manifold becomes a group manifold. In 1992, N. S. Agashe and M. R. Chafle [1], studied properties of semi-symmetric non-metric connection on Riemannian manifold and in 2017 S. Pahan, B. Pal, A. Bhattacharyya [14] studied multiply warped product on quasi-Einstein manifold with respect to semi-symmetric metric connection.

\section{Preliminaries}

A linear connection $\nabla$ on a Riemannian manifold $\left(M^{n}, g\right)$ is called metric connection if $\nabla g=0$, otherwise, it is called non-metric connection. A linear connection is symmetric metric connection iff it is Levi-Civita connection. A linear connection $\bar{\nabla}$ on $(M, g)$ is said to be semi-symmetric connection if its torsion tensor $T$ is of the form

$$
T(X, Y)=\pi(Y) X-\pi(X) Y,
$$

where $\pi$ is a 1 -form with the allied vector field $P$ defined by $\pi(X)=g(X, P)$, for any vector field $X$ on $M$.

The relation between semi-symmetric metric connection $\bar{\nabla}$ and the Levi-Civita connection $\nabla$ on $M$ is given by

$$
\bar{\nabla}_{X} Y=\nabla_{X} Y+\pi(Y) X-g(X, Y) P
$$

for each smooth vector fields $X$ and $Y$ on $M$. Further, a relation between the curvature tensors $R$ and $\bar{R}$ of type $(1,3)$ of the connections $\nabla$ and $\bar{\nabla}$ respectively is given by

$$
\begin{aligned}
\bar{R}(X, Y) Z= & R(X, Y) Z+g\left(Z, \nabla_{X} P\right) Y-g\left(Z, \nabla_{Y} P\right) X \\
& +g(X, Z)\left[\nabla_{Y} P+\pi(P) Y-\pi(Y) P\right] \\
& -g(Y, Z)\left[\nabla_{X} P+\pi(P) X-\pi(X) P\right] \\
& +\pi(Z)[\pi(Y) X-\pi(X) Y]
\end{aligned}
$$

for each smooth vector fields $X, Y$ and $Z$ on $M$.

Also the Ricci tensor and scalar tensor with respect to the semi-symmetric metric connection $\bar{\nabla}$ are, respectively, as follows:

$$
\overline{\operatorname{Ric}}(X, Y)=\sum_{i=1}^{n} g\left(\bar{R}\left(E_{i}, X\right) Y, E_{i}\right)
$$

and

$$
\bar{S}=\sum_{i=1}^{n} \overline{\operatorname{Ric}}\left(E_{i}, E_{i}\right)
$$

where $\left\{E_{1}, \ldots, E_{n}\right\}$ is a frame field on $M$.

Notation: Throught this paper, we will consider the followings:

(1) $M=B \times \times_{f} F, \operatorname{dim}(B)=n_{1}, \operatorname{dim}(F)=n_{2}, \operatorname{dim}(M)=n_{1}+n_{2}=\bar{n}$.

(2) Ric denotes the Ricci curvature on $M=B \times{ }_{f} F$. 
(3) $\operatorname{Ric}^{B}$ and $\operatorname{Ric}^{F}$ denote the lifts to $M$ of the Ricci curvature of $B$ and $F$ respectively, and $\overline{R i c}^{B}$ and $\overline{R i c}^{F}$ denote the lifts to $M$ of the Ricci curvature of $B$ and $F$ with respect to the semi-symmetric metric connection $\bar{\nabla}$, respectively.

(4) $R i c_{B}$ and $R i c_{F}$ denotes the Ricci curvature of $B$ and $F$ respectively, $\overline{R i c}_{B}$ and $\overline{R i c}_{F}$ denotes the Ricci curvature of $B$ and $F$ with respect to the semi-symmetric metric connection $\bar{\nabla}$ respectively.

(5) $\overline{d i v}, \overline{R i c}$ and $\bar{H}^{f}$ represent divergence, Ricci and Hessian of $f$ with respect to $\bar{\nabla}$ respectively.

(6) $\left|\nabla_{B} f\right|_{B}^{2}=g\left(\nabla_{B} f, \nabla_{B} f\right)$.

Now from Proposition 3.5 of [15], we have the following theorem.

Theorem 2.1. Let $M=B \times_{f} F$ be a warped product, $\operatorname{dim}(B)=n_{1}, \operatorname{dim}(F)=n_{2}$, $\operatorname{dim}(M)=n_{1}+n_{2}=\bar{n}$. If $X, Y \in \Gamma(T B), V, W \in \Gamma(T F)$ and $P \in \Gamma(T B)$, then

$$
\begin{aligned}
\overline{\operatorname{Ric}}(X, Y)= & \overline{\operatorname{Ric}}^{B}(X, Y)-n_{2}\left[\frac{H_{B}^{f}(X, Y)}{f}+\left\{\frac{P f}{f}+\pi(P)\right\} g(X, Y)\right. \\
& \left.+g\left(Y, \nabla_{X} P\right)-\pi(X) \pi(Y)\right]
\end{aligned}
$$

(2.1.2) $\overline{\operatorname{Ric}}(X, V)=\overline{\operatorname{Ric}}(V, X)=0$,

$$
\begin{aligned}
\overline{\operatorname{Ric}}(V, W)= & \overline{\operatorname{Ric}}^{F}(V, W)-\left\{\frac{\Delta_{B} f}{f}+\left(n_{2}-1\right) \frac{\left|\nabla_{B} f\right|_{B}^{2}}{f^{2}}+(\bar{n}-2) \pi(P)\right. \\
& \left.+\operatorname{div}_{B} P+\left(\bar{n}+n_{2}-2\right) \frac{P f}{f}\right\} g(V, W)
\end{aligned}
$$

where $\operatorname{div}(P)=\sum_{k=1}^{n_{1}}\left\langle\nabla_{E_{k}} P, E_{k}\right\rangle$, and $E_{k}, 1 \leq k \leq n_{1}$ is an orthonormal basis of $B$.

From the above theorem, we get the necessary and sufficient condition for warped product $M=B \times{ }_{f} F$ to be an Einstein manifold with respect to semi-symmetric metric connection $\bar{\nabla}$.

Corollary 2.2. The warped product $M=B \times_{f} F$ with Ric $=\lambda g$ is Einstein if and only if the following conditions hold:

(2.2.1) $\overline{\operatorname{Ric}}_{B}(X, Y)=\left[\lambda+n_{2} \frac{P f}{f}+n_{2} \pi(P)\right] g_{B}(X, Y)$

$$
+n_{2}\left[\frac{H_{B}^{f}(X, Y)}{f}+g_{B}\left(Y, \nabla_{X} P\right)-\pi(X) \pi(Y)\right]
$$

(2.2.2) $\left(F, g_{F}\right)$ is Einstein with $\overline{\operatorname{Ric}}_{F}(X, Y)=\lambda^{\prime} g_{F}(X, Y)$, for every $X, Y \in \Gamma(T B)$ and $V, W \in \Gamma(T F)$.

$(2.2 .3) \lambda^{\prime}=\lambda f^{2}+f \Delta_{B} f+\left(n_{2}-1\right)\left|\nabla_{B} f\right|^{2}+(\bar{n}-2) f^{2} \pi(P)+f^{2} d i v_{B} P+\left(\bar{n}+n_{2}-2\right) f P f$.

\section{Curvature, Ricci and scalar tensor with respect to the semi-symmetric metric connection}

In (2.2), (2.3) and (2.4), we have seen the expression for the curvature, Ricci and scalar tensor with respect to the semi-symmetric metric connection respectively.

We know that, if $(M, g)$ is a Riemannian manifold with Levi-civita connection $\nabla$ and $Z$ is a gradient vector field on $M$, then

$$
g\left(X, \nabla_{Y} Z\right)=g\left(Y, \nabla_{X} Z\right)
$$

for every smooth vector fields $X$ and $Y$ on $M$.

Now using this result, we prove the following lemma. 
Lemma 3.1. Let $(M, g)$ be a Riemannian manifold with Levi-civita connction $\nabla$ and $P$ is a gradient vector field on $(M, g)$, then

$$
g\left(X, \nabla_{P} P\right)=\left(\nabla_{P} \pi\right)(X)=\frac{1}{2} d(\pi(P))(X),
$$

for every smooth vector field $X$ on $M$.

Proof. Since $\nabla$ is Levi-Civita connection therefore

$$
\begin{aligned}
0 & =\left(\nabla_{P} g\right)(X, P) \\
& =\left(\nabla_{P} \pi\right)(X)-g\left(X, \nabla_{P} P\right),
\end{aligned}
$$

and

$$
\begin{aligned}
0 & =\left(\nabla_{X} g\right)(P, P) \\
& =\nabla_{X} \pi(P)-2 g\left(P, \nabla_{X} P\right) .
\end{aligned}
$$

As $P$ is a gradient vector field thus the result follows.

Using $g(\bar{R}(X, Y) Z, W)=\bar{R}(X, Y, Z, W)$, we prove the following propositions.

Proposition 3.2. Let $(M, g)$ be a Riemannian manifold with semi-symmetric metric connection $\bar{\nabla}$, then

(3.2.1) $\bar{R}(X, Y) Z=-\bar{R}(Y, X) Z$.

(3.2.2) $\bar{R}(X, Y, Z, W)=-\bar{R}(Y, X, Z, W)$.

(3.2.3) $\bar{R}(X, Y, Z, W)=-\bar{R}(X, Y, W, Z)$.

(3.2.4) If $P$ is gradient vector field then

(i) $\bar{R}(X, Y) Z+\bar{R}(Y, Z) X+\bar{R}(Z, X) Y=0$.

(ii) $\bar{R}(X, Y, Z, W)+\bar{R}(Y, Z, X, W)+\bar{R}(Z, X, Y, W)=0$.

(iii) $\bar{R}(X, Y, Z, W)=\bar{R}(Z, W, X, Y)$.

Proof. The proof of (3.2.1) is straightforward from the definition of curvature tensor in (2.2) and the second part will follow immediate from the first part. After some manipulation we can also prove (3.2.3). Next, we will prove the first part of (3.2.4)

$$
\begin{aligned}
\bar{R}(X, Y) Z+ & \bar{R}(Y, Z) X+\bar{R}(Z, X) Y=R(X, Y) Z+R(Y, Z) X+R(Z, X) Y \\
& +g\left(X, \nabla_{Y} P\right) Z-g\left(X, \nabla_{Z} P\right) Y+g\left(Y, \nabla_{Z} P\right) X \\
& -g\left(Y, \nabla_{X} P\right) Z+g\left(Z, \nabla_{X} P\right) Y-g\left(Z, \nabla_{Y} P\right) X .
\end{aligned}
$$

From the Bianchi's first identity and equation (3.1), we have R.H.S. of the above equation will be zero. The second and third part of (3.2.4) follows from the first part of (3.2.4).

Proposition 3.3. Let $\left(B^{n_{1}}, g_{B}\right)$ be a Riemannian manifold and $\left\{E_{1}, \ldots, E_{n_{1}}\right\}$ is a frame field on $B$, then

(3.3.1) $\operatorname{Ric}_{B}(X, Y)=\operatorname{Ric}_{B}(X, Y)+\left(2-n_{1}\right) g_{B}\left(Y, \nabla_{X} P\right)+[-\operatorname{div} P$

$$
\left.+\left(2-n_{1}\right) \pi(P)\right] g_{B}(X, Y)+\left(n_{1}-2\right) \pi(X) \pi(Y),
$$

for every smooth vector fields $X$ and $Y$ on $B$.

(3.3.2) $\bar{S}_{B}=S_{B}+\left(n_{1}-1\right)\left[\left(2-n_{1}\right) \pi(P)-2 \operatorname{div} P\right]$.

Proof. If $X$ and $Y$ are any smooth vector fields on $B$, then

$$
\overline{\operatorname{Ric}}_{B}(X, Y)=\sum_{i=1}^{n} g_{B}\left(\bar{R}\left(E_{i}, X\right) Y, E_{i}\right)
$$

Using (2.2) in the above equation and some manipulations provide (3.3.1). Next,

$$
\bar{S}_{B}=\sum_{i=1}^{n} \overline{\operatorname{Ric}}_{B}\left(E_{i}, E_{i}\right)
$$

Using (3.3.1) in the above equation provides (3.3.2). 
Remark. $\overline{\operatorname{Ric}}_{B}$ is symmetric (0,2)-type tensor if and only if $P$ is gradient vector field.

Proposition 3.4. Let $\left(B^{n_{1}}, g_{B}\right)$ be a Riemannian manifold and $\overline{R i c}_{B}$ is the symmetric $(0,2)$-type tensor then for each smooth vector fields $X$ on $B$

(3.4.1) $\overline{\operatorname{div}}\left(\overline{\operatorname{Ric}}_{B}\right)(X)=\operatorname{div}\left(\operatorname{Ric}_{B}\right)(X)-S_{B} \pi(X)-d(\operatorname{div} P)(X)+n_{1} \operatorname{Ric}_{B}(X, P)$

$$
\begin{aligned}
& +\left(2-n_{1}\right) g\left(X, \sum_{i=1}^{n_{1}} \nabla_{E_{i}, E_{i}}^{2} P\right)+\frac{\left[\left(2+2 n_{1}-n_{1}^{2}\right)\right]}{2} d(\pi(P))(X) \\
& +2\left(2 n_{1}-3\right)(\operatorname{div} P) \pi(X)+\left(1-n_{1}\right)\left(2-n_{1}\right) \pi(P) \pi(X) .
\end{aligned}
$$

$(3.4 .2) \operatorname{div}\left(\overline{\operatorname{Ric}}_{B}\right)(X)=\operatorname{div}\left(\operatorname{Ric}_{B}\right)(X)-d(\operatorname{div} P)(X)+\left(n_{1}-2\right)(\operatorname{div} P) \pi(X)$

$$
+\left(2-n_{1}\right) g\left(X, \sum_{i=1}^{n_{1}} \nabla_{E_{i}, E_{i}}^{2} P\right)+\frac{\left(2-n_{1}\right)}{2} d(\pi(P))(X) .
$$

Proof. Let $\left\{E_{1}, \ldots, E_{n_{1}}\right\}$ is a frame field on $B . \overline{\operatorname{Ric}}_{B}$ is symmetric $(0,2)$-type tensor therefore

$$
\begin{aligned}
\overline{\operatorname{div}}\left(\overline{\operatorname{Ric}}_{B}\right)(X) & =\sum_{i=1}^{n_{1}}\left(\bar{\nabla}_{E_{i}} \overline{\operatorname{Ric}}_{B}\right)\left(E_{i}, X\right) \\
& =\sum_{i=1}^{n_{1}} \bar{\nabla}_{E_{i}}\left(\overline{\operatorname{Ric}}_{B}\left(E_{i}, X\right)\right)-\sum_{i=1}^{n_{1}} \overline{\operatorname{Ric}}_{B}\left(\bar{\nabla}_{E_{i}} E_{i}, X\right)-\sum_{i=1}^{n_{1}} \overline{\operatorname{Ric}}_{B}\left(E_{i}, \bar{\nabla}_{E_{i}} X\right) .
\end{aligned}
$$

Now, we calculate the value of all terms of the R.H.S. of the above equation

$$
\begin{aligned}
\sum_{i=1}^{n_{1}} \bar{\nabla}_{E_{i}}\left(\overline{R i c}_{B}\left(E_{i}, X\right)=\right. & \sum_{i=1}^{n_{1}} \nabla_{E_{i}}\left(\operatorname{Ric}_{B}\left(E_{i}, X\right)\right)-d(\operatorname{div} P)(X) \\
& +\left(2-n_{1}\right) \sum_{i=1}^{n_{1}} \nabla_{E_{i}}\left(g\left(X, \nabla_{E_{i}} P\right)\right)+\left[\left(2-n_{1}\right) \pi(P)-\operatorname{div} P\right] \\
& \times \sum_{i=1}^{n_{1}} \nabla_{E_{i}}\left(g\left(E_{i}, X\right)\right)+\left(2-n_{1}\right) d(\pi(P))(X) \\
+ & \left(n_{1}-2\right)\left\{d(\pi(X))(P)+\pi(X) \sum_{i=1}^{n_{1}} \nabla_{E_{i}}\left(\pi\left(E_{i}\right)\right)\right\} \\
\sum_{i=1}^{n_{1}} \overline{R i c}_{B}\left(\bar{\nabla}_{E_{i}} E_{i}, X\right)= & \sum_{i=1}^{n_{1}} \operatorname{Ric} c_{B}\left(\nabla_{E_{i}} E_{i}, X\right)+\left(2-n_{1}\right) \sum_{i=1}^{n_{1}} g\left(X, \nabla_{\nabla_{i}} E_{i} P\right) \\
& +\left[\left(2-n_{1}\right) \pi(P)-\operatorname{div} P\right] \sum_{i=1}^{n_{1}} g\left(\nabla_{E_{i}} E_{i}, X\right) \\
& +\left(n_{1}-2\right) \pi(X) \sum_{i=1}^{n_{1}} \pi\left(\nabla_{E_{i}} E_{i}\right)+\left(1-n_{1}\right)\left[\operatorname{Ric}_{B}(X, P)\right. \\
& +\operatorname{div} P \pi(X)]+\left(2-3 n_{1}+n_{1}^{2}\right) g\left(X, \nabla_{P} P\right),
\end{aligned}
$$




$$
\begin{aligned}
\sum_{i=1}^{n_{1}} \overline{\operatorname{Ric}}_{B}\left(E_{i}, \bar{\nabla}_{E_{i}} X\right)= & \sum_{i=1}^{n_{1}} \operatorname{Ric}_{B}\left(E_{i}, \nabla_{E_{i}} X\right)+S_{B} \pi(X)-\operatorname{Ric}_{B}(X, P) \\
& +\left(2-n_{1}\right) \sum_{i=1}^{n_{1}} g\left(\nabla_{E_{i}} X, \nabla_{E_{i}} P\right)+\left[\left(2-n_{1}\right) \pi(P)-\operatorname{div} P\right] \\
& \times \sum_{i=1}^{n_{1}} g\left(E_{i}, \nabla_{E_{i}} X\right)+\left(3-2 n_{1}\right) \operatorname{div} P \pi(X)+\left(n_{1}-2\right) \\
& \times\left[\pi\left(\nabla_{P} X\right)+\pi\left(\nabla_{X} P\right)\right]+\left(n_{1}-1\right)\left(2-n_{1}\right) \pi(P) \pi(X) .
\end{aligned}
$$

Now, after substituting the value of $(3.3),(3.4)$, and (3.5) in the expression of $\overline{\operatorname{div}}\left(\overline{\operatorname{Ric}}_{B}\right)(X)$ and using (3.2) we get the first part. Proof of the second part is same as above.

\section{Second order semi-symmetric metric connection}

Let $(M, g)$ be a Riemanniam manifold with semi-symmetric metric connection $\bar{\nabla}$. Let $T$ be a $(r, s)$ - type tensor field. The second order semi-symmetric metric connection derivative of $T$ denoted by $\bar{\nabla}^{2} T$ is a $(r, s+2)-$ type tensor field and

$$
\begin{aligned}
\left(\bar{\nabla}_{X, Y}^{2} T\right)\left(\theta^{1}, \ldots, \theta^{r}, Z_{1}, \ldots, Z_{s}\right)= & \left(\bar{\nabla}_{X}(\bar{\nabla} T)\right)\left(Y, \theta^{1}, \ldots, \theta^{r}, Z_{1}, \ldots, Z_{s}\right) \\
= & \left.\bar{\nabla}_{X}\left(\bar{\nabla}_{Y} T\right)\right)\left(\theta^{1}, \ldots, \theta^{r}, Z_{1}, \ldots, Z_{s}\right) \\
& -\left(\bar{\nabla}_{\bar{\nabla}_{X} Y} T\right)\left(\theta^{1}, \ldots, \theta^{r}, Z_{1}, \ldots, Z_{s}\right)
\end{aligned}
$$

From the above, we have the followings:

(i) If $f: M \rightarrow \mathbb{R}$ is a smooth function then the second order semi-symmetric metric tensor derivative of $f$ with respect to $X$ and $Y$ is

$$
\begin{aligned}
\bar{\nabla}_{X, Y}^{2} f & =\left(\bar{\nabla}_{X} \bar{\nabla} f\right)(Y) \\
& =\left(\bar{\nabla}_{X} d f\right)(Y) \\
& =\nabla_{X, Y}^{2} f-\pi(Y) X f+g(X, Y) P f
\end{aligned}
$$

where we use the fact that $\bar{\nabla} f=d f$ in the second line.

(ii) If $X, Y$ and $Z$ are smooth vector fields on $(M, g)$ then second order semi-symmetric metric tensor derivative of $Z$ with respect to $X$ and $Y$ is

$$
\begin{aligned}
\bar{\nabla}_{X, Y}^{2} Z= & \bar{\nabla}_{X} \bar{\nabla}_{Y} Z-\bar{\nabla}_{\bar{\nabla}_{X} Y} Z \\
= & \nabla_{X, Y}^{2} Z+\left(\nabla_{X} \pi(Z)\right) Y+\pi\left(\nabla_{Y} Z\right) X-\pi(Y) \nabla_{X} Z \\
& +g(X, Y)\left[\nabla_{P} Z-\pi(Z) P\right]+g(Y, Z)\left[-\nabla_{X} P-\pi(P) X+\pi(X) P\right] \\
& +g(X, Z) \pi(Y) P-\left[g\left(X, \nabla_{Y} Z\right)-g\left(Y, \nabla_{X} Z\right)\right] P .
\end{aligned}
$$

(iii)

$$
\begin{aligned}
\bar{\nabla}_{X, Y}^{2} Z-\bar{\nabla}_{Y, X}^{2} Z= & R(X, Y) Z+g\left(Z, \nabla_{X} P\right) Y-g\left(Z, \nabla_{Y} P\right) X+\pi(X) \nabla_{Y} Z \\
& -\pi(Y) \nabla_{X} Z+g(X, Z)\left[\nabla_{Y} P+\pi(P) Y\right] \\
& -g(Y, Z)\left[\nabla_{X} P+\pi(P) X\right]
\end{aligned}
$$


Lemma 4.1. Let $X$ and $Y$ be smooth vector fields on Riemannian manifold $M$. If $w$ is a first form and $\bar{\nabla}$ is a semi-symmetric metric connection on $M$ then

$$
\begin{aligned}
& \left(\bar{\nabla}_{X} w\right)^{\#}=\nabla_{X} w^{\#}-w(X) P+w(P) X \\
& \left(\bar{\nabla}_{X, Y}^{2} w\right)^{\#}=\nabla_{X, Y}^{2} w^{\#}-\nabla_{X}(w(Y) P)-\left(\left(\nabla_{Y} w\right)(X)\right) P-\pi(Y) \nabla_{X} w^{\#} \\
& +w\left(\nabla_{X} Y\right) P+\nabla_{X}(w(P) Y)+\left(\left(\nabla_{Y} w\right)(P)\right) X+g(X, Y)\left[\nabla_{P} w^{\#}-w(P) P\right] \\
& -w(P) \nabla_{X} Y+w(Y)[\pi(X) P-\pi(P) X]+\pi(Y)[w(X) P-w(P) X] \\
& \left(\bar{\nabla}_{X, Y}^{2} w\right)(Z)=\left(\nabla_{X, Y}^{2} w\right)(Z)-\pi(Z)\left(\nabla_{X} w\right)(Y)-w(Y)\left(\nabla_{X} \pi\right)(Z) \\
& -\pi(Z)\left(\nabla_{Y} w\right)(X)-\pi(Y)\left(\nabla_{X} w\right)(Z)+g(X, Y)\left[\left(\nabla_{P} w\right)(Z)\right. \\
& -\pi(Z) w(P)]+g(X, Z)\left[\left(\nabla_{Y} w\right)(P)-\pi(P) w(Y)\right] \\
& \left.+g(Y, Z)\left(\nabla_{X} w(P)\right)\right]+[\pi(X) w(Y)+\pi(Y) w(X)] \pi(Z) \\
& \left(\bar{\nabla}_{X, Y}^{2} w\right)(Z)-\left(\bar{\nabla}_{Y, X}^{2} w\right)(Z)=-w(R(X, Y) Z)+\pi(X)\left(\nabla_{Y} w\right)(Z) \\
& -\pi(Y)\left(\nabla_{X} w\right)(Z)+w(X)\left(\nabla_{Y} \pi\right)(Z)-w(Y)\left(\nabla_{X} \pi\right)(Z) \\
& -g(X, Z)\left[w\left(\nabla_{Y} P\right)+\pi(P) w(Y)\right]+g(Y, Z)\left[w\left(\nabla_{X} P\right)+\pi(P) w(X)\right]
\end{aligned}
$$

where \# is musically operator and $\bar{\nabla}_{X, Y}^{2}=\bar{\nabla}_{X} \bar{\nabla}_{Y}-\bar{\nabla}_{\bar{\nabla}_{X} Y}$ denote the second order semisymmetric metric connection on $M$.

Proof. Let $w$ stands for the dual 1-form associated to $w^{\#}$ that is, we know that if $w$ is a first form then $w^{\#}$ is a vector field corresponding to $w$ and we can define

$$
w(X)=g\left(w^{\#}, X\right),
$$

for any smooth vector fields $X$ on $B$.

From (4.4), we deduce the following results

$$
\left(\nabla_{X} w\right)^{\#}=\nabla_{X} w^{\#}
$$

i.e.,

$$
g\left(\nabla_{X} w^{\#}, Y\right)=\left(\nabla_{X} w\right)(Z)
$$

and

$$
\left(\nabla_{X, Y}^{2} w\right)^{\#}=\nabla_{X, Y}^{2} w^{\#}
$$

Recall that

$$
\left(\nabla_{X} \pi\right)(Z)=g\left(\nabla_{X} P, Z\right) .
$$

The proof of (4.1.1) goes as follows:

$$
\begin{aligned}
\left(\bar{\nabla}_{X} w\right)(Y) & =\bar{\nabla}_{X} w(Y)-w\left(\bar{\nabla}_{X} Y\right) \\
& =\left(\nabla_{X} w\right)(Y)-\pi(Y) w(X)+g(X, Y) w(P) \\
& =g\left(\nabla_{X} w^{\#}, Y\right)-g(w(X) P, Y)+g(w(P) X, Y) \\
& =g\left(\nabla_{X} w^{\#}-w(X) P+w(P) X, Y\right),
\end{aligned}
$$

where we have used (4.6) in the third line. Therefore from (4.5) the last equation implies that

$$
\left(\bar{\nabla}_{X} w\right)^{\#}=\nabla_{X} w^{\#}-w(X) P+w(P) X .
$$

Hence (4.1.1) is proved.

Now, we prove the second part of the lemma. Since \# is a linear operator we have

$$
\left(\bar{\nabla}_{X, Y}^{2} w\right)^{\#}=\left(\bar{\nabla}_{X}\left(\bar{\nabla}_{Y} w\right)\right)^{\#}-\left(\bar{\nabla}_{\bar{\nabla}_{X} Y} w\right)^{\#},
$$


Applying (4.1.1) to both terms of the right hand side of (4.9), we have

$$
\begin{aligned}
\left(\bar{\nabla}_{X}\left(\bar{\nabla}_{Y} w\right)\right)^{\#}= & \nabla_{X}\left(\nabla_{Y} w^{\#}\right)-\nabla_{X}(w(Y) P)-\left(\left(\nabla_{Y} w\right)(X)\right) P \\
& +\nabla_{X}(w(P) Y)+\left(\left(\nabla_{Y} w\right)(P)\right) X+w(P)[\pi(Y) X \\
& -g(X, Y) P]+w(Y)[\pi(X) P-\pi(P) X]
\end{aligned}
$$

and

$$
\begin{aligned}
\left(\bar{\nabla}_{\bar{\nabla}_{X} Y} w\right)^{\#}= & \nabla_{\nabla_{X} Y} w^{\#}+\pi(Y) \nabla_{X} w^{\#}-w\left(\nabla_{X} Y\right) P+w(P) \nabla_{X} Y \\
& -g(X, Y) \nabla_{P} w^{\#}+\pi(Y)[w(P) X-w(X) P] .
\end{aligned}
$$

After putting (4.10) and (4.11) in (4.9) we get the result (4.1.2).

Next, we prove the third part of the lemma. We can write

$$
\left(\bar{\nabla}_{X, Y}^{2} w\right)(Z)=g\left(\left(\bar{\nabla}_{X, Y}^{2} w\right)^{\#}, Z\right) .
$$

After using the value of $\left(\bar{\nabla}_{X, Y}^{2} w\right)$ \# from (4.1.2), and applying (4.6) and (4.8) to the last equation we get the result (4.1.3). Hence third part of lemma is proved. The proof of the last part of the lemma follows immediately from (4.1.3).

Remark. The result (4.1.4) of Lemma 4.1 is the expression of Ricci identity [8, p. 159] with respect to the semi-symmetric metric connection.

\section{Hessian of $f$ with respect to semi-symmetric metric connection}

Definition 5.1. Let $(M, g)$ be Riemannian manifold of $\operatorname{dim} n$. Then, Hessian of a smooth function $f: M \rightarrow \mathbb{R}$ with respect to the semi-symmetric metric connection $\bar{\nabla}$ is denoted by $\bar{H}^{f}$ and defined by $\bar{H}^{f}:=\bar{\nabla}(\bar{\nabla} f)$

Lemma 5.2. The Hessian $\bar{H}^{f}$ of $f$ is a (0,2)-type tensor such that

$$
\bar{H}^{f}(X, Y)=H^{f}(X, Y)-\pi(Y) X f+g(X, Y) P f
$$

for every smooth vector fields $X$ and $Y$ on $M$.

Proof. Since $\bar{\nabla} f=d f$ we have

$$
\bar{H}^{f}(X, Y)=\bar{\nabla}(d f)(X, Y)=\left(\bar{\nabla}_{X} d f\right)(Y) .
$$

Then the proof follows from (4.2).

Remark. The Hessian $\bar{H}^{f}$ is a symmetric (0,2)-type tensor if and only if

$$
\pi(Y) X f=\pi(X) Y f
$$

for any smooth vector fields $X$ and $Y$ on $M$.

Lemma 5.3. If $\bar{H}^{f}$ is a symmetric (0,2)-type tensor then

$$
\pi(X)|\nabla f|^{2}=d f(P) d f(X),
$$

for any smooth vector field $X$ on $M$.

Proof. Let $X$ be a smooth vector field on $M$. Then

$$
\begin{aligned}
\pi(X)|\nabla f|^{2} & =\pi(X) g(\nabla f, \nabla f) \\
& =\pi(X) d f(\nabla f) \\
& \stackrel{(5.2)}{=} \pi(\nabla f) d f(X) \\
& =d f(P) d f(X) .
\end{aligned}
$$


Lemma 5.4. Let $(M, g)$ be a Riemannian manifold and $f: M \rightarrow \mathbb{R}$ be a smooth function. If $\bar{H}^{f}$ is symmetric then

(5.4.1) $\left(\bar{\nabla}_{X} d f\right)(Y)=\left(\bar{\nabla}_{Y} d f\right)(X)$,

(5.4.2) $\left(\bar{\nabla}_{X, Y}^{2} d f\right)(Z)=\left(\bar{\nabla}_{X, Z}^{2} d f\right)(Y)$,

for every smooth vector fields $X, Y$ and $Z$ on $M$.

Proof. From definition of $\bar{H}^{f}$, we have

$$
\left(\bar{\nabla}_{X} d f\right)(Y)=\bar{H}^{f}(X, Y)
$$

Hence, the symmetry of $\bar{H}^{f}$ proves the first part. Now, we prove the second part. Let us write

$$
\text { L.H.S }=\bar{\nabla}_{X}\left(\bar{\nabla}_{Y} d f(Z)\right)-\left(\bar{\nabla}_{Y} d f\right)\left(\bar{\nabla}_{X} Z\right)-\left(\bar{\nabla}_{\bar{\nabla}_{X} Y} d f\right)(Z)
$$

After using (5.4.1) in this equation, we get

$$
=\bar{\nabla}_{X}\left(\bar{\nabla}_{Z} d f(Y)\right)-\left(\bar{\nabla}_{\bar{\nabla}_{X} Z} d f\right)(Y)-\left(\bar{\nabla}_{Z} d f\right)\left(\bar{\nabla}_{X} Y\right)=\text { R.H.S. }
$$

Proposition 5.5. Let $\left(B^{n_{1}}, g_{B}\right)$ be a Riemannian manifold. If $\bar{H}^{f}$ is a symmetric $(0,2)$-type tensor then for every smooth vector fields $X$ on $B$ :

(5.5.1) $\overline{\operatorname{div}}\left(\bar{H}^{f}\right)(X)=\operatorname{div}\left(H^{f}\right)(X)-\left(\nabla_{\nabla f} \pi\right)(X)+d(P f)(X)-2 \Delta f \pi(X)$

$$
+n_{1} H^{f}(X, P)+\left(1-n_{1}\right) \pi(P) d f(X) .
$$

(5.5.2) $\operatorname{div}\left(\bar{H}^{f}\right)(X)=\operatorname{div}\left(H^{f}\right)(X)+\left(\nabla_{\nabla} \pi\right)(X)-\Delta f \pi(X)+d(P f)(X)$.

(5.5.3) $\overline{\operatorname{div}}\left(\frac{1}{f} \bar{H}^{f}\right)(X)=\operatorname{div}\left(\frac{1}{f} H^{f}\right)(X)+\frac{1}{f}\left\{d(P f)(X)-\left(\nabla_{\nabla f} \pi\right)(X)-2 \Delta f \pi(X)\right.$

$$
\left.+n_{1} H^{f}(X, P)+\left(1-n_{1}\right) \pi(P) d f(X)\right\}
$$

(5.5.4) $\operatorname{div}\left(\frac{1}{f} \bar{H}^{f}\right)(X)=\operatorname{div}\left(\frac{1}{f} H^{f}\right)(f)+\frac{1}{f}\left\{d(P f)(X)-\nabla f \pi(X)-\left(\nabla_{\nabla f} \pi\right)(X)\right\}$.

Proof. Let $\left\{E_{1}, \ldots, E_{n_{1}}\right\}$ be a frame field on $B$. Since $\bar{H}^{f}$ is symmetric $(0,2)$-type tensor, we get

$$
\begin{aligned}
\overline{\operatorname{div}}\left(\bar{H}^{f}\right)(X) & =\sum_{i=1}^{n_{1}}\left(\bar{\nabla}_{E_{i}} \bar{H}^{f}\right)\left(E_{i}, X\right) \\
& =\sum_{i=1}^{n_{1}} \bar{\nabla}_{E_{i}}\left(\bar{H}^{f}\left(E_{i}, X\right)\right)-\sum_{i=1}^{n_{1}} \bar{H}^{f}\left(\bar{\nabla}_{E_{i}} E_{i}, X\right)-\sum_{i=1}^{n_{1}} \bar{H}^{f}\left(E_{i}, \bar{\nabla}_{E_{i}} X\right)
\end{aligned}
$$


Now, we need to calculate the value of all terms of the R.H.S. of the above equation:

$$
\begin{aligned}
\sum_{i=1}^{n_{1}} \bar{\nabla}_{E_{i}}\left(\bar{H}^{f}\left(E_{i}, X\right)\right)= & \sum_{i=1}^{n_{1}} \nabla_{E_{i}}\left(H^{f}\left(E_{i}, X\right)\right)-\nabla_{\nabla f}(\pi(X))+d(P f)(X) \\
& -\pi(X) \sum_{i=1}^{n_{1}} \nabla_{E_{i}} \nabla_{E_{i}} f+(P f) \sum_{i=1}^{n_{1}} \nabla_{E_{i}} g\left(E_{i}, X\right), \\
\sum_{i=1}^{n_{1}} \bar{H}^{f}\left(\bar{\nabla}_{E_{i}} E_{i}, X\right)= & \sum_{i=1}^{n_{1}} \bar{H}^{f}\left(\nabla_{E_{i}} E_{i}, X\right)-\pi(X) \sum_{i=1}^{n_{1}} \nabla_{\nabla_{E_{i}} E_{i}} f \\
& +(P f) \sum_{i=1}^{n_{1}} g_{B}\left(\nabla_{E_{i}} E_{i}, X\right)+\left(1-n_{1}\right) H^{f}(X, P), \\
\sum_{i=1}^{n_{1}} \bar{H}^{f}\left(E_{i}, \bar{\nabla}_{E_{i}} X\right)= & \left.\sum_{i=1}^{n_{1}} H^{f}\left(E_{i}, \nabla_{E_{i}} X\right)+\pi(X) \Delta f-\pi\left(\nabla_{\nabla f} X\right)\right)-H^{f}(X, P) \\
& +(P f) \sum_{i=1}^{n_{1}} g_{B}\left(E_{i}, \nabla_{E_{i}} X\right)+\left(n_{1}-1\right) \pi(P) d f(X) .
\end{aligned}
$$

After introducing (5.5), (5.6) and (5.7) in (5.4) we get the result (5.5.1).

The proof of (5.5.2) is the same as the above. Next, we prove (5.5.3). We have

$$
\begin{aligned}
\overline{\operatorname{div}}\left(\frac{1}{f} \bar{H}^{f}\right)(X) & =\sum_{i=1}^{n_{1}}\left(\bar{\nabla}_{E_{i}}\left(\frac{1}{f} \bar{H}^{f}\right)\right)\left(E_{i}, X\right) \\
= & \sum_{i=1}^{n_{1}} \bar{\nabla}_{E_{i}}\left(\frac{1}{f} \bar{H}^{f}\left(E_{i}, X\right)\right)-\frac{1}{f} \sum_{i=1}^{n_{1}} \bar{H}^{f}\left(\bar{\nabla}_{E_{i}} E_{i}, X\right)-\frac{1}{f} \sum_{i=1}^{n_{1}} \bar{H}^{f}\left(E_{i}, \bar{\nabla}_{E_{i}} X\right),
\end{aligned}
$$

and

$$
\begin{aligned}
\sum_{i=1}^{n_{1}} \bar{\nabla}_{E_{i}}\left(\frac{1}{f} \bar{H}^{f}\left(E_{i}, X\right)\right)= & \sum_{i=1}^{n_{1}} \nabla_{E_{i}}\left(\frac{1}{f} H^{f}\left(E_{i}, X\right)\right)+\frac{1}{f}\left\{-\nabla_{\nabla f}(\pi(X))+d(P f)(X)\right. \\
& \left.-\pi(X) \sum_{i=1}^{n_{1}} \nabla_{E_{i}} \nabla_{E_{i}} f+(P f) \sum_{i=1}^{n_{1}} \nabla_{E_{i}}\left(g\left(E_{i}, P\right)\right)\right\} .
\end{aligned}
$$

Therefore, substituting (5.9), (5.6) and (5.7) into (5.8) implies the result (5.5.3). The proof of (5.5.4). is similar to the above.

\section{Einstein warped product space with non positive scalar curvature with respect to semi-symmetric metric connection}

Lemma 6.1. Let $\left(B^{n_{1}}, g_{B}\right)$ be a Riemannian manifold with semi-symmetric metric connection $\bar{\nabla}$ and $f$ be a smooth function on $B$. If $\bar{H}^{f}$ is symmetric then for any smooth vector field $X$ on $B$ the following holds:

$$
\begin{aligned}
\operatorname{div}\left(H^{f}\right)(X)= & d(\Delta f)(X)+\operatorname{Ric}(\nabla f, X) \\
& +(\Delta f) \pi(X)-d(P f)(X) .
\end{aligned}
$$

Proof. Substituting $w=d f$ in (4.1.4) and using the result (5.4.2), we get

$$
\begin{aligned}
\left(\bar{\nabla}_{X, Z}^{2} d f\right)(Y)-\left(\bar{\nabla}_{Y, X}^{2} d f\right)(Z)= & -d f(R(X, Y) Z)+\pi(X)\left(\nabla_{Y} d f\right)(Z)-\pi(Y)\left(\nabla_{X} d f\right)(Z) \\
& +d f(X)\left(\nabla_{Y} \pi\right)(Z)-d f(Y)\left(\nabla_{X} \pi\right)(Z) \\
& -g(X, Z)\left[d f\left(\nabla_{Y} P\right)+\pi(P) d f(Y)\right] \\
& +g(Y, Z)\left[d f\left(\nabla_{X} P\right)+\pi(P) d f(X)\right],
\end{aligned}
$$

for any smooth vector fields $X, Y$ and $Z$ on $B$.

For a fixed $p \in B$, we can find a local orthonormal frame $\left\{E_{1}, \ldots E_{n_{1}}\right\}$ of the space $B$ such 
that $\nabla_{E_{i}} E_{i}(p)=0$. We can also choose $\nabla_{E_{i}} Y(p)=\nabla_{E_{i}} P(p)=0$. Taking trace of (6.2) with respect to $X, Z$ and from the symmetry of $\bar{H}^{f}$, we have

$$
\begin{aligned}
\sum_{i=1}^{n_{1}}\left(\bar{\nabla}_{E_{i}, E_{i}}^{2} d f\right)(Y)= & \sum_{i=1}^{n_{1}}\left(\bar{\nabla}_{Y, E_{i}}^{2} d f\right)\left(E_{i}\right)-\sum_{i=1}^{n_{1}} d f\left(R\left(E_{i}, Y\right) E_{i}\right) \\
& +d(P f)(Y)-(\Delta f) \pi(Y)+\left(1-n_{1}\right) \pi(P) d f(Y)
\end{aligned}
$$

Since,

$$
\begin{aligned}
\sum_{i=1}^{n_{1}}\left(\bar{\nabla}_{E_{i}, E_{i}}^{2} d f\right)(Y)= & \operatorname{div}\left(H^{f}\right)(Y)-2(\Delta f) \pi(Y) \\
& +\left(n_{1}+1\right) d(d f)(Y)+\left(1-n_{1}\right) \pi(P) d f(X)
\end{aligned}
$$

and

$$
\sum_{i=1}^{n_{1}}\left(\bar{\nabla}_{Y, E_{i}}^{2} d f\right)\left(E_{i}\right)=d(\Delta f)(Y)+\left(n_{1}-1\right) d(P f)(X) .
$$

Therefore after using $(6.4),(6.5)$ in $(6.3)$ we get the result (6.1). Hence proved the lemma.

Proposition 6.2. Let $\left(B^{n_{1}}, g_{B}\right)$ be a compact Riemannian manifold with semi-symmetric metric connection $\bar{\nabla}$ of dimension $n_{1}>2$, and both of $\bar{H}^{f}$ and $\overline{R i c}_{B}$ both be symmetric tensors. Let $f$ be a non-constant smooth function on $B$ satisfying (2.2.1) for a constant $\lambda \in \mathbb{R}$ and a natural number $n_{2} \in \mathbb{N}$. Then $f$ satisfies (2.2.3) for a constant $\lambda^{\prime}$ if

$$
\begin{aligned}
& a_{1}(P f)(X f)+f\left\{a_{2} d(P f)(X)+a_{3} d f\left(\nabla_{X} P\right)\right. \\
& \left.+a_{4} \pi(P) X f+2 \Delta f \pi(X)\right\}+f^{2}\left\{a_{5} d(\operatorname{div} P)(X)\right. \\
& \left.+a_{6} d(\pi(P))(X)+a_{7} g\left(X, \sum_{i=1}^{n_{1}} \nabla_{E_{i}, E_{i}}^{2} P\right)+a_{8} \operatorname{div} P \pi(X)\right\}=0,
\end{aligned}
$$

for every smooth vector field $X$ on $B$ and

$$
\begin{aligned}
& a_{1}=\left(n_{2}-n_{1}\right), a_{2}=-\left(\bar{n}+2 n_{1}-2\right), a_{3}=2(\bar{n}-2), a_{4}=-2 \bar{n}, \\
& a_{5}=\frac{2(2-\bar{n})}{n_{2}}, a_{6}=-\frac{(\bar{n}-2)^{2}}{n_{2}}, a_{7}=\frac{a_{3}}{n_{2}}, a_{8}=\frac{2(2-\bar{n})}{n_{2}} .
\end{aligned}
$$

Moreover, we can construct a compact Einstein warped product space $M=B \times_{f} F$ with Ric $=\lambda g$ for a compact Einstein space $\left(F, g_{F}\right)$ of dimension $n_{2}$ with $\overline{R i c}_{F}=\lambda^{\prime} g_{F}$.

Proof. On contracting both sides of (2.2.1), we have

$$
\bar{S}_{B}=n_{1}\left\{\lambda+n_{2} \frac{P f}{f}+n_{2} \pi(P)\right\}+n_{2}\left\{\frac{\Delta f}{f}+\operatorname{div} P-\pi(P)\right\} .
$$

The above equation implies that

$$
\begin{aligned}
d \bar{S}_{B}(X)= & n_{2}\left\{\left(n_{1}-1\right) d(\pi(P))(X)+d(\operatorname{div} P)(X)+\frac{1}{f} d(\Delta f)(X)\right. \\
& \left.+\frac{n_{1}}{f} d(P f)(X)-\frac{\left[n_{1}(P f)+\Delta f\right]}{f^{2}} d f(X)\right\}
\end{aligned}
$$

From equation (3.3.2), we have

$$
\begin{aligned}
d \bar{S}_{B}(X)= & 2 \operatorname{div}\left(\operatorname{Ric}_{B}\right)(X)+\left(n_{1}-1\right)\left[\left(2-n_{1}\right) d(\pi(P))(X)\right. \\
& -2 d(\operatorname{div} P)(X)] .
\end{aligned}
$$


From equation (6.7) and (6.8), we have

$$
\begin{aligned}
2 \operatorname{div}\left(\operatorname{Ric}_{B}\right)(X)= & \frac{n_{2}}{f} d(\Delta f)(X)+\frac{n_{1} n_{2}}{f} d(P f)(X)-\frac{n_{2}}{f^{2}} \Delta f d f(X) \\
& \left.-\frac{n_{1} n_{2}}{f^{2}} P f d f(X)+\left[\left(\bar{n}+n_{1}-2\right) \lambda_{2}\right)\right] d(\operatorname{div} P)(X) \\
& +(2-\bar{n})\left(1-n_{1}\right) d(\pi(P))(X) .
\end{aligned}
$$

The equation (2.2.1) can be written as

$$
\begin{aligned}
\overline{\operatorname{Ric}}_{B}(X, Y)= & {\left[\lambda+n_{2} \pi(P)\right] g_{B}(X, Y)+n_{2} g\left(Y, \nabla_{X} P\right) } \\
& -n_{2} \pi(X) \pi(Y)+\frac{n_{2}}{f} \pi(Y) d f(X)+\frac{n_{2}}{f} \bar{H}^{f}(X, Y) .
\end{aligned}
$$

Taking divergence on both sides of (6.10) and using (3.2), we get

$$
\begin{aligned}
\operatorname{div}\left(\overline{\operatorname{Ric}}_{B}\right)(X)= & n_{2} \operatorname{div}\left(\frac{1}{f} \bar{H}^{f}\right)(X)+n_{2} g\left(X, \sum_{i=1}^{n_{1}} \nabla_{E_{i}, E_{i}}^{2} P\right)-n_{2} \operatorname{div} P \pi(X) \\
& +\frac{n_{2}}{2} d(\pi(P))(X)+\frac{n_{2}}{f}\left(\nabla_{\nabla f} \pi\right)(X) \\
& +\frac{n_{2}}{f} \Delta f \pi(X)-\frac{n_{2}}{f^{2}}|\nabla f|^{2} \pi(X) .
\end{aligned}
$$

Using (3.4.2) and (5.5.4) in (6.11), we have

$$
\begin{aligned}
\operatorname{div}\left(\operatorname{Ric}_{B}\right)(X) & -n_{2} \operatorname{div}\left(\frac{1}{f} H^{f}\right)(X)-d(\operatorname{div} P)(X)+(\bar{n}-2) \operatorname{div}(P) \pi(X) \\
& +(2-\bar{n}) g_{B}\left(X, \sum_{i=1}^{n_{1}} \nabla_{E_{i}, E_{i}}^{2} P\right)+\frac{(2-\bar{n})}{2} d(\pi(P))(X) \\
& -\frac{n_{2}}{f} d(P f)(X)+\frac{n_{2}}{f^{2}} P f d f(X)=0 .
\end{aligned}
$$

Multiplying both sides of the above equation by $-\frac{2 f^{2}}{n_{2}}$ we have

$$
\begin{aligned}
2 f^{2} \operatorname{div}\left(\frac{1}{f} H^{f}\right)(X) & -\frac{2}{n_{2}} f^{2} \operatorname{div}\left(\operatorname{Ric}_{B}\right)(X)+\frac{2}{n_{2}} f^{2} d(\operatorname{div} P)(X)+2 f d(P f)(X) \\
& -2(P f) d f(X)+\frac{(\bar{n}-2) f^{2}}{n_{2}}\left\{2\left(X, \sum_{i=1}^{n_{1}} \nabla_{E_{i}, E_{i}}^{2} P\right)\right. \\
& +d(\pi(P))(X)-2 \operatorname{div}(P) \pi(X)\}=0 .
\end{aligned}
$$

From equations (2.2.1) and (3.3.1), we have

$$
\begin{aligned}
\operatorname{Ric}_{B}(X, Y)= & {\left[\lambda+\operatorname{div} P+\frac{n_{2}}{f} P f+(\bar{n}-2) \pi(P)\right] g_{B}(X, Y)+\frac{n_{2}}{f} H^{f}(X, Y) } \\
& +(\bar{n}-2) g_{B}\left(Y, \nabla_{X} P\right)+(2-\bar{n}) \pi(X) \pi(Y) .
\end{aligned}
$$

Also, we know that

$$
\operatorname{div}\left(\frac{1}{f} H^{f}\right)(X)=-\frac{1}{2 f^{2}} d\left(\left|\nabla_{B} f\right|_{B}^{2}\right)+\frac{1}{f} \operatorname{div}\left(H^{f}\right)(X) .
$$

Using Lemma 6.1 and from equation (6.14) the last equation implies

$$
\begin{aligned}
\operatorname{div}\left(\frac{1}{f} H^{f}\right)(X)= & \frac{\left(n_{2}-1\right)}{2 f^{2}} d\left(\left|\nabla_{B} f\right|_{B}^{2}\right)+\frac{n_{2}}{f^{2}}(P f) d f(X)+\frac{1}{f}\{d(\Delta f)(X) \\
& +\lambda d f(X)+\operatorname{div} P d f(X)+(\bar{n}-2) d f\left(\nabla_{X} P\right) \\
& +\Delta f \pi(X)-d(P f)(X)-2 \pi(P) d f(X)\} .
\end{aligned}
$$


Using the equations (6.15), (6.9) and condition (6.6) in (6.13), we get

$$
\begin{aligned}
d\left\{\lambda f^{2}\right. & +f \Delta f+\left(n_{2}-1\right) d\left(\left|\nabla_{B} f\right|_{B}^{2}\right\}(X)+(\bar{n}-2)\{2 f \pi(P) d f(X) \\
& \left.+f^{2} d(\pi(P))(X)\right\}+2 f d i v P d f(X)+f^{2} d(\operatorname{div})(X) \\
& +\left(\bar{n}+n_{2}-2\right)\{(P f) d f(X)+f d(P f)(X)\}=0 .
\end{aligned}
$$

This equation can be written as

$$
\begin{gathered}
d\left\{\lambda f^{2}+f \Delta_{B} f+\left(n_{2}-1\right)\left|\nabla_{B} f\right|^{2}+(\bar{n}-2) f^{2} \pi(P)\right. \\
\left.+f^{2} \lambda_{2} \operatorname{div}_{B} P+\left(\bar{n}+n_{2}-2\right) f(P f)\right\}=0
\end{gathered}
$$

Hence the first part of the proposition is proved. The second part of the proposition holds by using the sufficiencies of Corollary 2.2 .

Theorem 6.3. Let $M=B \times{ }_{f} F$ be an Einstein warped product space with semi-symmetric metric connection $\bar{\nabla}$ and compact base $B$. If $M$ has non-positive scalar curvature with $\operatorname{dim}(F)=n_{2}>2$ and warping function $f$ satisfies

$$
\begin{aligned}
\frac{\lambda_{2}}{V(B)} \int_{B}\left\{f^{2} \operatorname{div} P-f(x)^{2} \operatorname{div} P(x)\right\} & +\frac{b_{1}}{V(B)} \int_{B}\left\{f^{2} \pi(P)-f(x)^{2} \pi(P)(x)\right\} \\
& +\frac{b_{2}}{V(B)} \int_{B} f(P f)=0,
\end{aligned}
$$

where $x$ may be minimum or maximum points of $f$ on $B$ and $V(B)$ denotes the volume of $B, b_{1}=(\bar{n}-2), b_{2}=\left(\bar{n}+n_{2}-2\right)$, then $M$ is simply a Riemannian product.

Proof. Since $\operatorname{dim}(F)=n_{2}>2$ and $\operatorname{Ric}_{F}=\lambda^{\prime} g_{F_{i}}$ where

$$
\lambda^{\prime}=\lambda f^{2}+f \Delta_{B} f+\left(n_{2}-1\right)\left|\nabla_{B} f\right|^{2}+(\bar{n}-2) f^{2} \pi(P)+f^{2} d i v_{B} P+\left(\bar{n}+n_{2}-2\right) f P f
$$

then by $\left[4\right.$, Sec 3] $\lambda^{\prime}$ is constant.

Equation (2.2.3) can be written as

$$
\begin{aligned}
\lambda^{\prime}= & \lambda f^{2}+\operatorname{div}(f \nabla f)+\left(n_{2}-2\right)\left|\nabla_{B} f\right|^{2}+\left[(\bar{n}-1) \lambda_{1} \lambda_{2}-\lambda_{2}^{2}\right] f^{2} \pi(P) \\
& +\lambda_{2} f^{2} \operatorname{div}_{B} P+\left[(\bar{n}) \lambda_{1}+\left(n_{2}-1\right) \lambda_{2}\right] f P f .
\end{aligned}
$$

By taking integration of (6.17) over $B$, we have

$$
\begin{aligned}
\lambda^{\prime}= & \frac{\lambda}{V(B)} \int_{B} f^{2}+\frac{\left(n_{2}-2\right)}{V(B)} \int_{B}\left|\nabla_{B} f\right|^{2}+\frac{\lambda_{2}}{V(B)} \int_{B} f^{2} \operatorname{div}_{B} P \\
& +\frac{b_{1}}{V(B)} \int_{B} f^{2} \pi(P)+\frac{b_{2}}{V(B)} \int_{B} f(P f) .
\end{aligned}
$$

Case 1. Let $n_{2} \geq 3$ and $l$ be the maximum point of $f$ on $B$. Then we have $f(l)>0, \nabla f(l)=0$ and $\Delta f(l) \leq 0$. Therefore from (2.2.3) and (6.18), we have

$$
\begin{aligned}
0 \geq & f(l) \Delta f(l) \\
= & \lambda^{\prime}-\lambda f(l)^{2}-\lambda_{2} f(l)^{2} \operatorname{div} P(l)-b_{1} f(l)^{2} \pi(P)(l) \\
= & \frac{\lambda}{V(B)} \int_{B}\left\{f^{2}-f(l)^{2}\right\}+\frac{\left(n_{2}-2\right)}{V(B)} \int_{B}\left|\nabla_{B} f\right|^{2}+\frac{\lambda_{2}}{V(B)} \int_{B}\left\{f^{2} \operatorname{div}_{B} P\right. \\
& \left.-f(l)^{2} \operatorname{div} P(l)\right\}+\frac{b_{1}}{V(B)} \int_{B}\left\{f^{2} \pi(P)-f(l)^{2} \pi(P)(l)\right\}+\frac{b_{2}}{V(B)} \int_{B} f(P f) \\
\geq & 0 .
\end{aligned}
$$

The last inequality holds from the properties of $\lambda$ and by the condition (6.16). Hence $f$ is constant. 
Case 2. Let $n_{2}=1,2$ and we consider $m$ as a minimum point of $f$ on $B$. Then we have $f(m)>0, \nabla f(m)=0$ and $\Delta f(m) \geq 0$. Therefore from (2.2.3) and (6.18), we have

$$
\begin{aligned}
0 \leq & f(m) \Delta f(m) \\
= & \lambda^{\prime}-\lambda f(l)^{2}-\lambda_{2} f(m)^{2} \operatorname{div} P(m)-b_{1} f(l)^{2} \pi(P)(m) \\
= & \frac{\lambda}{V(B)} \int_{B}\left\{f^{2}-f(l)^{2}\right\}+\frac{\left(n_{2}-2\right)}{V(B)} \int_{B}\left|\nabla_{B} f\right|^{2}+\frac{\lambda_{2}}{V(B)} \int_{B}\left\{f^{2} \operatorname{div}_{B} P\right. \\
& \left.-f(m)^{2} \operatorname{div} P(m)\right\}+\frac{b_{1}}{V(B)} \int_{B}\left\{f^{2} \pi(P)-f(m)^{2} \pi(P)(m)\right\}+\frac{b_{2}}{V(B)} \int_{B} f(P f) \\
\leq & 0 .
\end{aligned}
$$

Therefore from the properties of $\lambda$ and condition (6.16), we can say that $f$ is constant. The result follows.

Acknowledgment. We would like to thank the referees for their valuable suggestions to improve the paper.

\section{References}

[1] N.S. Agashe and M.R. Chafle, A semi-symmetric non-metric connection in a Riemannian manifold, Indian J. Pure Appl. Math. 23 (6), 399-409, 1992.

[2] R. Bishop and B. O'Neill, Manifolds of negative curvature, Trans. Am. Math. Soc. 145, 1-49, 1969.

[3] A.L. Besse, Einstein manifolds. Ergeb. Math. Grenzgeb., 3. Folge, Bd. 10. Berlin, Heidelberg, New York, Springer-Verlag. 1987.

[4] F. Dobarro and B, Ünal, Curvature of multiply warped products, J. Geom. Phys. 55 (1), 75-106, 2005.

[5] D. Dumitru, On Compact Einstein Warped Products, Annals Of Spiru Haret University: Mathematics-Informatics Series, Bucharest, Romania, 2011.

[6] A. Friedmann and J.A. Schouten, Über die Geometrie der halbsymmetrischen Ubertragungen, Math. Z. 21, 211-223, 1924.

[7] F.E.S. Feitosa, A.A.F. Filho and J.N.V. Gomes, On the construction of gradient Ricci soliton warped product, Nonlinear Analysis, 161, 30-43, 2017.

[8] S. Gallot, D. Hulin and J. Lafontaine, Riemannian Geometry, Springer-Verlag, Berlin, 1987.

[9] H.A. Hayden, Subspace of a space with torsion, Proc. Lond. Math. Soc. II Series, 34, 27-50, 1932.

[10] D.S. Kim, Compact Einstein Warped Product Spaces, Trends Math. 5 (2), 1-5, 2002.

[11] D.S. Kim and Y.H. Kim, On compact Einstein warped product spaces with nonpositive scalar curvature, Proc. Amer. Math. Soc. 131 (8), 2573-2576, 2003.

[12] M.T. Mustafa, A non-existence result for compact Einstein warped products, J. Phys. A: Math. Gen. 38, L791-L793, 2005.

[13] B. O'Neill, Semi-Riemannian Geometry. With Applications to Relativity, Pure and Applied Mathematics 103, Academic Press, Inc., New York, 1983.

[14] S. Pahan, B. Pal and A. Bhattacharyya, Multiply warped product on quasi-Einstein manifold with a semi-symmetric metric connection, Analele Universitatii Oradea Fasc. Matematica XXIV (1), 171-183, 2017.

[15] Q. Qu and Y. Wang, Multiply warped products with a quarter-Symmetric connection, J. Math. Anal. Appl. 431 (2), 955-987, 2015.

[16] K. Yano, On semi-symmetric connection, Rev. Roumaine Math Pures App. 15, 15791586, 1970. 